\title{
Nigrostriatal dopaminergic control of operant and spatial behavior in the rat
}

\author{
D. C. UGURU-OKORIE \\ University of Ibadan, Ibadan, Nigeria
}

\begin{abstract}
Rats trained in a two-lever Skinner box to obtain food by leverpressing were given either a unilateral 6-OHDA lesion of the nigrostriatal bundle on the same side as or on the side opposite to the preferred forepaw or a control treatment, and were tested 1 week and 8 weeks after surgery. Only subjects in the contralaterally lesioned group reversed their forepaw preferences. Rate of leverpressing was depressed in all lesioned subjects, but more so in contralaterally lesioned ones. All lesioned subjects rotated mainly ipsiversively. Another group of rats given a unilateral kainate lesion of the striatum contralateral to the preferred forepaw and tested 1 week postoperatively displayed a reversal of forepaw preferences, a depression of leverpressing, and an increased preference for the lever opposite to the lesioned striatum. In a third experiment, rats trained to run a T-maze for food and then given a unilateral 6-OHDA lesion of the nigrostriatal bundle opposite to the preferred arm of the maze and tested 1 week later showed a reversal of side preferences. It is proposed that the nigrostriatal DA system is part of a lateralized system controlling forelimb use, rotational behavior, and side preference in a T-maze.
\end{abstract}

Since the classic experiments of Carlsson and his co-workers (Carlsson, Lindqvist, Magnusson, \& Waldeck, 1958; Carlsson \& Waldeck, 1958), which placed dopamine (DA) clearly on the map as a CNS event worth investigating in its own right, this catecholamine has been implicated in a wide variety of behavioral functions.

One such function is the sensorimotor regulation of behavior. Disruptions of the sensorimotor control of the contralateral forepaw have been reported following a unilateral lesion of the pallidum (Levine, Ferguson, Kreinick, Gustafson, \& Schwartzbaum, 1971; Levine \& Schwarzbaum, 1973) or of the entopeduncular nucleus (Levine \& Schwarzbaum, 1973). Lesions of these areas interrupt the ascending nigrostriatal DA pathway (Moore, Bhatnager, \& Heller, 1971; Ungerstedt, 1971a) and cause a reduction of striatal DA (Anden, Dahlstrom, Fuxe, Larsson, Olson, \& Ungerstedt, 1966). Also, unilateral lesions of the nigrostriatal dopamine system make animals incapable of orienting to stimuli applied to the contralateral side (Ljungberg \& Ungerstedt, 1976; Marshall, Richardson, \& Teitelbaum, 1974), and such animals

This study was carried out in the laboratories of the M.R.C. Brain Metabolism Unit, Edinburgh, as part of a $\mathrm{PhD}$ project. The author wishes to acknowledge with deep gratitude the immense help received during the study from David M. Vowles, Head of the Department of Psychology, University of Edinburgh, from Gordon W. Arbuthnott, of the M.R.C. Brain Metabolism Unit, Edinburgh, and from their two departments. Professor Vowles's constant encouragement throughout the study is especially appreciated. Many thanks are also due Janet Panther of the Department of Psychology, University of Edinburgh, for kindly doing the secretarial work. display impairments in using the contralateral limbs to resist gravitational pull (Marshall et al., 1974).

In view of such findings, there is little wonder that the nigrostriatal pathway is widely assumed to be part of a lateralized system controlling the sensorimotor components of behavior. This notion is more probable if one takes into consideration the fact that this pathway is structurally related to the extrapyramidal system (Bedard, Larochelle, Parent, \& Poirier, 1969).

The nigrostriatal DA system has also been implicated in rotational behavior. Asymmetry between the nigrostriatal DA systems of the two hemispheres of the brain has been shown to cause rotation in the direction opposite to the more active nigrostriatal system, whether asymmetry is produced by unilateral electrical stimulation (Arbuthnott \& Crow, 1971; Arbuthnott \& Ungerstedt, 1970; Zimmerberg \& Glick, 1975) or by unilateral damage (Anden et al., 1966; Christie \& Crow, 1971; Ungerstedt, 1971a). Studies using a combination of unilateral interruption of the nigrostriatal pathway and systemic administration of amphetamine or apomorphine have further established that increased neostriatal DA receptor activity in one hemisphere leads to circling in the direction opposite to the more active striatum (Ungerstedt, 1971b).

A large unilateral electrolytic lesion of the locus coeruleus, which does not actually interrupt the nigrostriatal pathway, has, however, been shown to be associated with strong, although transient, contraversive rotation when the lesioned animals are given a systemic injection of amphetamine or apomorphine (Donaldson, Dolphin, Jenner, Marsden, \& Pycock, 
1976; Pycock, Donaldson, \& Marsden, 1976). What is interesting about these later studies is that an elevation of DA levels was observed in the ipsilateral striatum, and this would appear to support the view that the circling behavior observed was related to an asymmetry in DA receptor stimulation. In fact, that was exactly the conclusion reached by the authors following further work.

Other investigators have demonstrated that asymmetry between the nigrostriatal DA systems determines side preference in a T-maze. Thus, unilateral electrical stimulation of the caudate nucleus evokes contralateral side preference (Zimmerberg \& Glick, 1975), whereas a unilateral caudate lesion produces ipsiversive side preference (Rothman \& Glick, 1976). Moreover, a correlation between rats' side preference in a T-maze and their direction of circling following D-amphetamine administration has been reported (Zimmerberg, Glick, \& Jerussi, 1974). It has, therefore, been proposed that side preference in a T-maze is determined by the relative dominance of one or the other nigrostriatal DA system of the brain (Zimmerberg, 1975; Zimmerberg et al., 1974).

This paper presents three experiments. Experiment 1 investigated the effects of a unilateral 6-OHDA lesion of the nigrostriatal system on the sensorimotor control of the forelimbs, rate of leverpressing in a foodrewarded operant-behavior situation, lever preference in a two-lever Skinner box, and rotational behavior. In this experiment, sets of experimental and control subjects were observed in terms of these variables before and after surgery. In Experiment 2, the nigrostriatal DA system was disrupted postsynaptically through intrastriatal administration of kainic acid, and the effects of this operation on the variables studied in Experiment 1 were observed. Experiment 3 investigated the effects of a unilateral nigrostriatal lesion (6-OHDA induced) on T-maze behavior, with particular reference to side and goal preferences. In this experiment, also, the subjects were observed both before and after surgery.

\section{EXPERIMENT 1}

\section{Materials and Methods}

Subjects. The subjects were 29 male Wistar albino rats weighing between 175 and $200 \mathrm{~g}$ at the time of surgery. Throughout the study, the animals were kept two per cage in $\mathrm{RCl}$ cages of North Kent Plastic Cages Ltd (measurements: $56 \times 38 \times 18 \mathrm{~cm}$ ) in a reversedaylight room. The subjects had unlimited access to food and water except that (1) they were deprived of food for predetermined periods of time at specific points in the study to prepare them for operant behavior sessions, (2) they had no water during operant behavior sessions, and (3) they had to work in the Skinner box for all the food they received during periods of data-gathering operant behavior sessions.

Behavioral studies. At the end of a 7-day disturbance-free orientation period in the laboratory housing conditions, the subjects were deprived of food for $48 \mathrm{~h}$, although water remained available. Following food deprivation, the subjects were trained to press a lever for food in a two-lever Skinner box. The subjects were free to operate either lever, and a subject received, for every push that depressed the lever, a single 45-mg food pellet (Campden Instruments, London).

After a subject reached the learning criterion of 20 leverpresses, followed immediately by the retrieval of food at the food-tray and, $24 \mathrm{~h}$ later, demonstrated retention of the operant reponse, it was passed as trained and ready for data-gathering sessions. Over the next 2 days, the subjects were given two recorded (i.e., data-gathering) sessions of $25 \mathrm{~min}$ each-one session per day. Then the animals underwent surgery.

Following surgery, the subjects were left undisturbed for 7 days before postoperative testing was started. At the end of the 1-week period, the subjects were deprived of food for $24 \mathrm{~h}$ in preparation for the early postoperative data-gathering sessions; two such sessions, lasting $25 \mathrm{~min}$ each, were given, one session per day, on 2 consecutive days. Thereafter, all behavioral studies were suspended until 8 weeks had elapsed from the day of surgery, to allow for complete degeneration of the nigrostriatal DA pathway and the occurrence of any associated compensatory events. Then, after another 24-h period of preparatory food deprivation, the subjects were given two "retest" (late postoperative) sessions on 2 consecutive days, each session lasting $25 \mathrm{~min}$, as usual.

The variables observed and recorded in the data-gathering sessions were: number of effective (i.e., rewarded) leverpresses executed with each forepaw; total number of effective presses; number of times each lever was effectively operated; and number of rotations in each direction (left/right). Recording of quantitative observations was carried out with the combined help of a manually operated multiple-channel counting device, each kind of observation (such as number of effective presses made on the left lever with the right forepaw) being recorded on one channel. The records obtained manually with respect to number of effective presses made on each lever, and so also with respect to total number of effective presses made, were checked against figures provided by a set of electromagnetic counters.

The recording of number of rotations in each direction was not automated because no tool was available for recording rotations without first strapping the subjects across the shoulders. It was thought that binding a subject across the shoulders could interfere with its leverpressing and other activities in the Skinner box. The pattern of forepaw use, as reflected in the number of presses made with each forepaw, was also not recorded automatically because an appropriate tool for automatic recording of such variables was not available.

Surgery. Following pretreatment with an ip injection of desipramine $(25 \mathrm{mg} / \mathrm{kg})$ and pargyline $(50 \mathrm{mg} / \mathrm{kg})$, the animals were anesthetized with a saturated Fluothane/air mixture and maintained under anesthesia by the circulation of about a $1 \%$ concentration of this substance through a mask fitted over the nose for the duration of surgery. The operation was carried out on a David Kopf stereotaxic instrument. A sagittal knife-cut was made in the skin of the animal's head and, after the required area of the skull was scraped clean, a hole about $2 \mathrm{~mm}$ in diameter was made with a dental drill. The membranes covering the cortex were then removed with care. A 30-ga stainless steel cannula attached to a Hamilton syringe was lowered through the hole into the medial forebrain bundle (MFB) at the following coordinates: $4.1 \mathrm{~mm}$ behind the bregma, $1.1 \mathrm{~mm}$ lateral to the midline, and $7.6 \mathrm{~mm}$ below the cortical surface. 6-OHDA hydrochloride was dissolved in isotonic saline that contained $.1 \mathrm{mg} / \mathrm{ml} \mathrm{L-ascorbic} \mathrm{acid.} \mathrm{Twenty-}$ three rats originally intended to serve as experimental subjects were given a unilateral microinjection of 6-OHDA (8 $\mu \mathrm{g}$ in $4 \mu \mathrm{l})$. Of this number, 13 subjects were injected on the side opposite to the forepaw used for most of the preoperative leverpresses, and the other 10 subjects were injected on the same side as the preoperative forepaw preference. Six primary control subjects were injected with the bare vehicle $(4 \mu \mathrm{l}$ of isotonic saline containing $.1 \mathrm{mg} / \mathrm{ml}$ 
L-ascorbic acid) on the side opposite to the preoperatively preferred forepaw; they did not receive 6-OHDA with it. Any 6OHDA-treated subjects in which the MFB target was missed were to serve as secondary control subjects.

At the end of each injection, $4 \mathrm{~min}$ were allowed to expire before the cannula was removed, the hole made in the skull in the process of surgery was covered with bone-wax, and the wound was given an antiseptic spray and sutured.

Verification of lesion. All the subjects in this experiment were sacrificed between 3 and 6 weeks after the late postoperative data-gathering sessions in the Skinner box. The animals were killed by being stunned and then decapitated. Left and right striatal samples were dissected out by making a coronal knife-cut through the brain at the middle of the optic chiasma, separating out the two halves of the rostral piece, and then exposing the ventral aspect of the striatum by carefully removing the septum and other tissues ventral to the striatum. Mesolimbic samples were also dissected out and included the nucleus accumbens, olfactory tubercle, and frontal cortex. The samples were used for the radioenzymatic determination of DA and NE contents of the brain regions sampled. Assay of these catecholamines was by the method of Coyle and Henry (1973) and Palkovitz, Brownstein, Saavedra, and Axelrod (1974). The striatal levels of DA in the two hemispheres would show the extent of nigrostriatal dopaminergic damage, while the striatal NE levels and the mesolimbic levels of DA and NE would indicate any unintended damage to catecholamine systems supplying the forebrain.

\section{Results}

Behavior in the Skinner box. All nine of the subjects with 6-OHDA destruction of the nigrostriatal DA system contralateral to the preoperatively preferred forepaw stopped pressing the lever mainly with that paw and tended to more often use the preoperatively nonpreferred forepaw when tested 1 week after surgery. At this stage, the overall picture was little more than that of a shift from the preoperative forepaw preference; at the retest 8 weeks after surgery, however, eight of the nine contralaterally lesioned subjects had completely reversed their forepaw preferences- to the extent, in fact, of using virtually only the preoperatively nonpreferred forepaw. The ninth subject showed a clear recovery of the preoperative forepaw preference at the retest carried out 8 weeks after surgery.

Eight subjects with 6-OHDA destruction of the nigrostriatal DA system ipsilateral to the preoperatively preferred forepaw retained their forepaw preferences when tested 1 week or 8 weeks after surgery.

Six partially lesioned subjects, whose 6-OHDA had practically missed the MFB target, retained their preoperative forepaw preferences irrespective of whether the neurotoxin was administered on the same side as or on the side opposite to the forepaw preferred prior to surgery.

The six primary control subjects, which had been injected contralaterally with the saline/ascorbic-acid vehicle not containing 6-OHDA, also retained their preoperative forepaw preferences when tested 1 and 8 weeks after surgery.

In Figure 1 are presented the mean patterns of effective forepaw use exhibited by the four different groups of subjects preoperatively, 1 week postoperatively, and 8 weeks postoperatively (Figure 1A).

All subjects with total or near-total unilateral nigrostriatal lesion showed a drop in the rate of leverpressing when tested 1 week after surgery. The drop was statistically significant at the .005 level in the case of the nine contralaterally lesioned subjects (Wilcoxon test, one-tailed) and at the .025 level in the case of the ipsilaterally lesioned subjects (Wilcoxon test, one-tailed). On the other hand, both the partially lesioned and the vehicle-injected control groups displayed an increase in the rate of leverpressing when tested 1 week postoperatively, although the increase was not statistically significant.

When retested 8 weeks after surgery, all four groups of subjects leverpressed more than they had at the 1-week postoperative test, although the group with a contralateral lesion still performed, on the average, fewer effective leverpresses than they had preoperatively over an identical period of time. Rates of leverpressing are also depicted in Figure 1B in terms of the mean number of effective (rewarded) leverpresses executed by each group of subjects in $50 \mathrm{~min}$ (two 25-min sessions). In the figure are presented the data from the preoperative and the two postoperative two-session runs.

Unilateral nigrostriatal lesion did not consistently determine lever preference in the present experiment. Thus, the group of subjects lesioned on the same side as the preoperatively preferred forepaw did not show a significant difference between the number of effective presses executed on the ipsilateral and the contralateral levers preoperatively, 1 week postoperatively, or 8 weeks postoperatively, although the group lesioned on the side opposite to the preoperatively preferred forepaw showed a significant preference for the ipsilateral lever at the 1 -week postoperative test $(p<.005$, Wilcoxon test, one-tailed), returning to the preoperative degree of difference (not significant) when tested 8 weeks after surgery. The unlesioned and partially lesioned groups of control subjects, for their part, had a significant preference for the ipsilateral lever prior to surgery $(p=$ .025 , Wilcoxon test, one-tailed) and retained this preference at the postoperative test $(p=.025$, Wilcoxon test, one-tailed). Figure 1 also furnishes a graphical comparison between the mean number of effective presses executed on the ipsilateral and contralateral levers by each group of subjects in $50 \mathrm{~min}$ (two 25-min sessions) preoperatively, 1 week postoperatively, and 8 weeks postoperatively (Figure 1C).

Prior to surgery, none of the four groups of subjects showed a clear preference for the left or right direction of spontaneous circling. However, at the test carried out 1 week after surgery, the two groups with a total or near-total unilateral lesion displayed a huge enhancement of spontaneous circling and rotated 
A

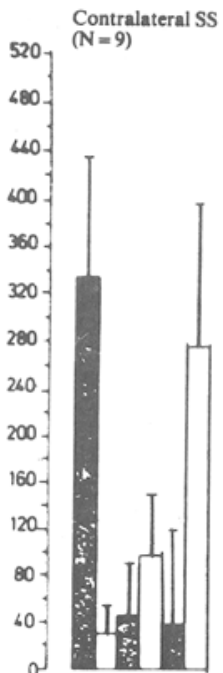

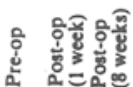

Ipsilateral SS

$$
\begin{aligned}
& \text { Ipsilateral } \\
& (\mathrm{N}=8)
\end{aligned}
$$

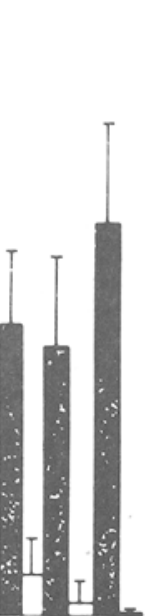

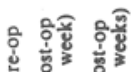

Control SS I wrongly placed 6 -OHDA) $\mathrm{N}=6$ )
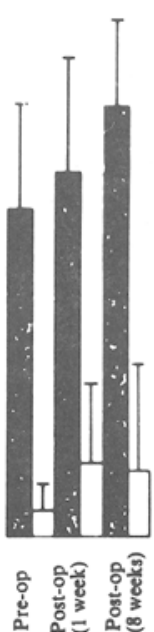

Control SS II

(ascorbic acid)

$(\mathrm{N}=6)$

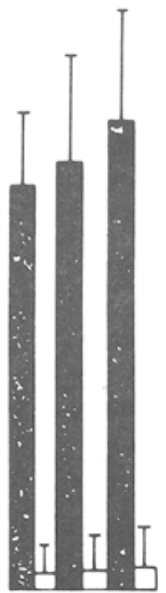

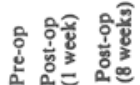

C

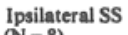
$(\mathrm{N}=8)$

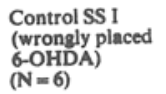

Control SS II

(vehicle-injected) Contral
$(\mathrm{N}=9)$ $(N=8)$

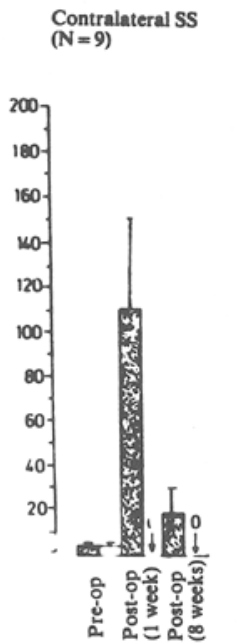

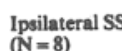

$(\mathrm{N}=8)$

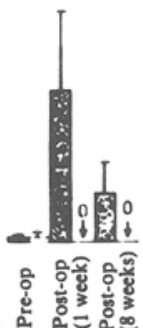

B

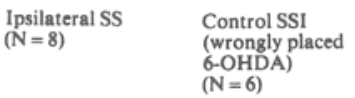

Control SS II

(ascorbic acid) $(\mathrm{N}=6)$
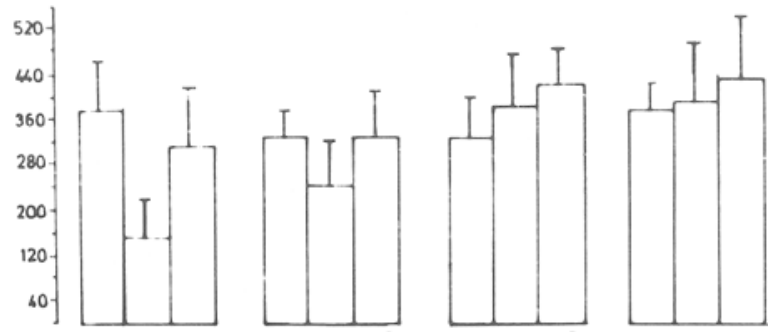

突

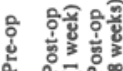

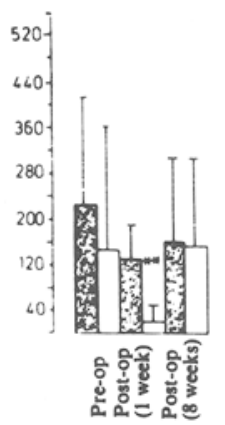
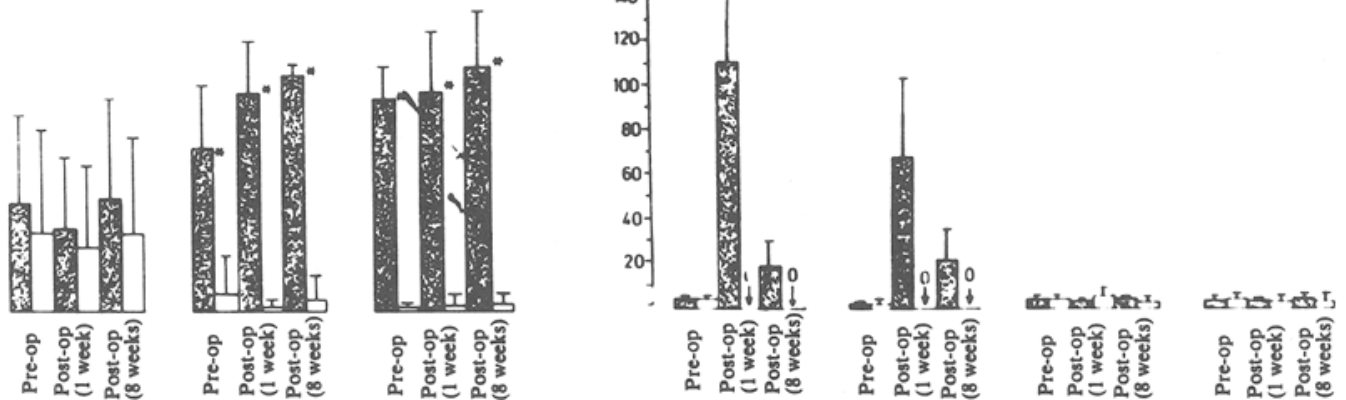

Figure 1. Mean forepaw preferences (A) (stippled columns, preoperatively preferred paw), leverpresses (B), lever choice (C) (stippled columns, ipsilateral lever), and rotations (D) (stippled columns, ipsiversive rotations) displayed by the 6-0HDA-lesioned and control groups of rats in 50 min of testing in a two-lever Skinner box, before surgery, 1 week after surgery, and 8 weeks after surgery. Each bar represents one SD. (Abbreviations: Pre-op, before surgery; Post-op, after surgery.)

exclusively in the direction of the lesion (ipsiversive circling). At the retest, 8 weeks after surgery, both lesioned groups were still circling more or less exclusively toward the lesioned side; however, the number of spontaneous rotations performed over an identical period of time $(50 \mathrm{~min})$ was much less than it was at the 1-week postoperative test.
Neither the control group of subjects sustaining a partial 6-OHDA lesion of the nigrostriatal DA system on one side nor the vehicle-injected control group displayed a statistically significant enhancement of the number of rotations or directional preference at the postoperative tests. Figure 1D shows the mean numbers of ipsiversive and contraversive rotations 
displayed by the four groups in 50 min prior to surgery, 1 week after surgery, and 8 weeks after surgery.

Catecholamine assay results. Table 1 shows the percent depletions of DA and NE in the various brain regions sampled in each subject.

There was more than $94 \%$ depletion of DA in the striatum on the lesion side in every one of the experimental subjects (ipsilaterally and contralaterally lesioned subjects alike). However, as the table shows, there were also reductions of striatal NE and of mesolimbic area DA and NE in these animals.

The 6-OHDA-injected control group also showed a certain amount of drop in DA and NE contents of the striatum and mesolimbic area on the injected side. The vehicle-injected control subjects, on the other hand, had no consistent difference between the two sides in catecholamine contents of the striatum and the mesolimbic area.

Table 1

Percent Catecholamine Loss in the Injected Hemisphere of Rats Given @ Unilateral 6-OHDA Microinjection into the MFB (Ipsilateral or Contralateral to the Predominantly Used Forepaw) and of Partially Lesioned and Vehicle-Treated Controls

\begin{tabular}{|c|c|c|c|c|}
\hline \multirow[b]{2}{*}{ Subject } & \multicolumn{2}{|c|}{ Dopamine } & \multicolumn{2}{|c|}{ Norepinephrine } \\
\hline & Striatum & Mesolimbic & Striatum & Mesolimbic \\
\hline
\end{tabular}

Contralaterally Lesioned (Experimental) Group

$\begin{array}{lrrrr}\text { A } & 99 & 91 & 99 & 0 \\ \text { B } & 100 & 100 & 98 & 65 \\ \text { C } & 100 & 98 & 95 & 23 \\ \text { D } & 97 & 100 & 78 & 17 \\ \text { E } & 96 & 94 & 72 & 47 \\ \text { F } & 99 & 99 & 93 & 15 \\ \text { G } & 100 & 100 & 89 & 33 \\ \text { H } & 100 & 100 & 89 & 33 \\ \text { I } & 98 & 70 & 96 & 75\end{array}$

Ipsilaterally Lesioned (Experimental) Group

$\begin{array}{lrrrr}\text { A } & 100 & 99 & 64 & 32 \\ \text { B } & 99 & 80 & 95 & 77 \\ \text { C } & 97 & 100 & 87 & 92 \\ \text { D } & 94 & 85 & 93 & 11 \\ \text { E } & 97 & 97 & 70 & 67 \\ \text { F } & 99 & 94 & 82 & 31 \\ \text { G } & 100 & 100 & 65 & 0 \\ \text { H } & 99 & 100 & 78 & 0\end{array}$

Partially Lesioned (Control) Group

$\begin{array}{lrrrr}\text { A } & 69 & 58 & 48 & 0 \\ \text { B } & 2 & 0 & 1 & 0 \\ \text { C } & 11 & 7 & 20 & 20 \\ \text { D } & 24 & 0 & 20 & 9 \\ \text { E } & 47 & 32 & 57 & 0 \\ \text { F } & 66 & 62 & 75 & 77\end{array}$

Vehicle-Injected (Control) Group

\begin{tabular}{lrrrr} 
A & 0 & 7 & 9 & 7 \\
B & 4 & 0 & 0 & 0 \\
C & 0 & 4 & 0 & 0 \\
D & 0 & 25 & 0 & 0 \\
E & 0 & 3 & 0 & 0 \\
F & 30 & 9 & 76 & 0 \\
\hline
\end{tabular}

\section{EXPERIMENT 2}

\section{Materials and Methods}

Subjects. The subjects for the present experiment were seven male Wistar albino rats weighing $180-200 \mathrm{~g}$ at the time of surgery. Throughout the experiment, the subjects were housed in twos in a reverse-daylight room (as in Experiment 1). The subjects had unrestricted access to food and water except during operant behavior sessions. When they were being prepared for a period of operant behavior sessions, they were deprived of food for predetermined periods of time, and, during the period itself, they had to work for all the food they received. Moreover, they had no access to water during a food-rewarded operant behavior session.

Behavioral studies. After an initial 7-day period of disturbancefree orientation in the laboratory housing conditions, the subjects were deprived of food for $48 \mathrm{~h}$ in preparation for training on a food-rewarded operant task. After training, the subjects were given a 25-min data-gathering session in the Skinner box daily for 2 successive days. Then the subjects underwent surgery. An interval of 7 days followed before postoperative testing in the operant behavior box was begun. There were, altogether, two 25 -min datagathering sessions, given one session per day on 2 successive days, in the postoperative stage of the experiment.

The variables observed and recorded in data-gathering sessions were: number of effective leverpresses made with each forepaw; total number of effective leverpresses, including those made with both forepaws; number of effective presses made on each lever; and number of rotations made in each direction. Procedures for recording quantitative observations were the same as those employed in Experiment 1.

Surgery. The subjects were anesthetized with a Fluothane/air mixture and operated upon by the same general surgical procedure used in Experiment 1. The coordinates used for microinjection of kainic acid into the striatum were: $.5 \mathrm{~mm}$ to the front of the bregma, $2.5 \mathrm{~mm}$ lateral to the midline, and $4.5 \mathrm{~mm}$ below the cortical surface. Kainic acid was dissolved in isotonic saline and adjusted to a $\mathrm{pH}$ of 7.4 with sodium hydroxide. Injection was done manually with the help of a Hamilton syringe, and each subject received $1.5 \mu \mathrm{g}$ of kainic acid in $.3 \mu \mathrm{l}$ of the vehicle solution over a 7-min period. At the end of each injection, $5 \mathrm{~min}$ were allowed to elapse before the injection cannula was removed. Then the hole in the skull was covered with bone wax, and the wound was given an antibacterial spray and sutured.

Dissection. Six weeks after surgery, the animals were sacrificed for the verification of lesion. After being stunned with a blow, they were decapitated and their brains were quickly removed. Striatal and mesolimbic samples were dissected out by the same procedure as that used in Experiment 1 except that the coronal knife-cut used to obtain these samples was placed at the rostral edge of the optic chiasma. Left and right nigral samples were also dissected out by making a coronal knife-cut at the level of the mammillary bodies and another just $1 \mathrm{~mm}$ behind; the substantia nigra carried in the left and right halves of the slab of tissue bounded by the coronal cuts was identified and carefully picked after a further cut just ventral and parallel to the medial lemniscus. The block of tissue bounded by the coronal knife-cuts at the mammillary bodies and just rostral to the optic chiasma constituted the specimen for use in the histological check on damage to nonstriatal tissues, notably the pallidum, that might have resulted from the diffusion of kainic acid from the injection site.

Blochemistry. Immediately after each striatal sample was dissected out and before it was stored in liquid nitrogen, it was crushed up and divided into two parts for separate biochemical assays of choline acetyltransferase (CAT) and glutamic acid decarboxylase (GAD) activities. The nigral sample from each side of the brain was used for GAD assay only. CAT was determined by a modification of the method of Fonnum (1975), and GAD was assayed using a combination of the methods of Drummond and 
Philips (1974) and Urquhart, Perry, Hansen, and Kennedy (1975). The mesolimbic sample from each hemisphere was used for the determination of DA and NE concentrations according to the radioenzymatic method of Coyle and Henry (1973) and Palkovitz et al. (1974).

Histology. Following dissection, the tissue specimen for histological examination was mounted on a disk of cork and frozen quickly with the help of solid carbon dioxide. Twenty-micron sections were cut with the help of a cryostat, and one in every five sections was picked. The sections were stained with luxol fast blue (for fibers) and cresyl fast violet (for cell bodies), according to a modification of the method of Kluver and Barrera (1953). The sections were mounted with DPX and viewed under a light microscope.

\section{Results}

Behavior in the Skinner box. Kainic acid lesion of the striatum contralateral to the forepaw used for most of the preoperative leverpresses caused all the subjects to display a clear reversal of forepaw preferences at the test carried out 1 week after surgery. The patterns of forepaw use in the execution of effective leverpresses before and after surgery are presented in Figure 2A.

Rate of leverpressing was depressed by the lesion. The mean number of rewarded leverpresses performed in identical time before and after surgery can be seen graphically represented in Figure 2B.

Unilateral striatal damage appears also to have affected lever choice. Thus, prior to surgery, the subjects operated the ipsilateral lever more times than the contralateral lever, but at the postoperative test the reverse was the case. Figure $2 \mathrm{C}$ depicts the mean number of presses executed on each lever pre- and postoperatively.

Spontaneous rotation, as recorded during operant behavior sessions in the Skinner box, was enhanced following surgery (see Figure 2D). Postoperative rotation was predominantly ipsiversive to the lesioned striatum. It should be noted that contraversive rotation also occurred, but, whereas postoperatively there was a significant difference $(p=.025$, Wilcoxon test, one-tailed) between the number of ipsiversive vs. contraversive rotations observed during the operant behavior sessions, preoperative records showed no significant difference between the two directions of circling.

Biochemical assay results. Table 2 shows that there were reductions of CAT and GAD activities in the lesioned striatum in all the subjects. Activity drop was not consistently greater in the case of either enzyme; in other words, some subjects showed a larger drop in striatal CAT than in striatal GAD, while for some others the reverse was the case. However, taken together, the combined activities of these enzymes suffered a drop of $50 \%$ or more in all the subjects except one, which had a $49 \%$ drop.

Nigral GAD activity was not markedly reduced. In fact, two subjects had a slightly higher GAD activity in the substantia nigra of the lesioned side than in the substantia nigra of the opposite side.
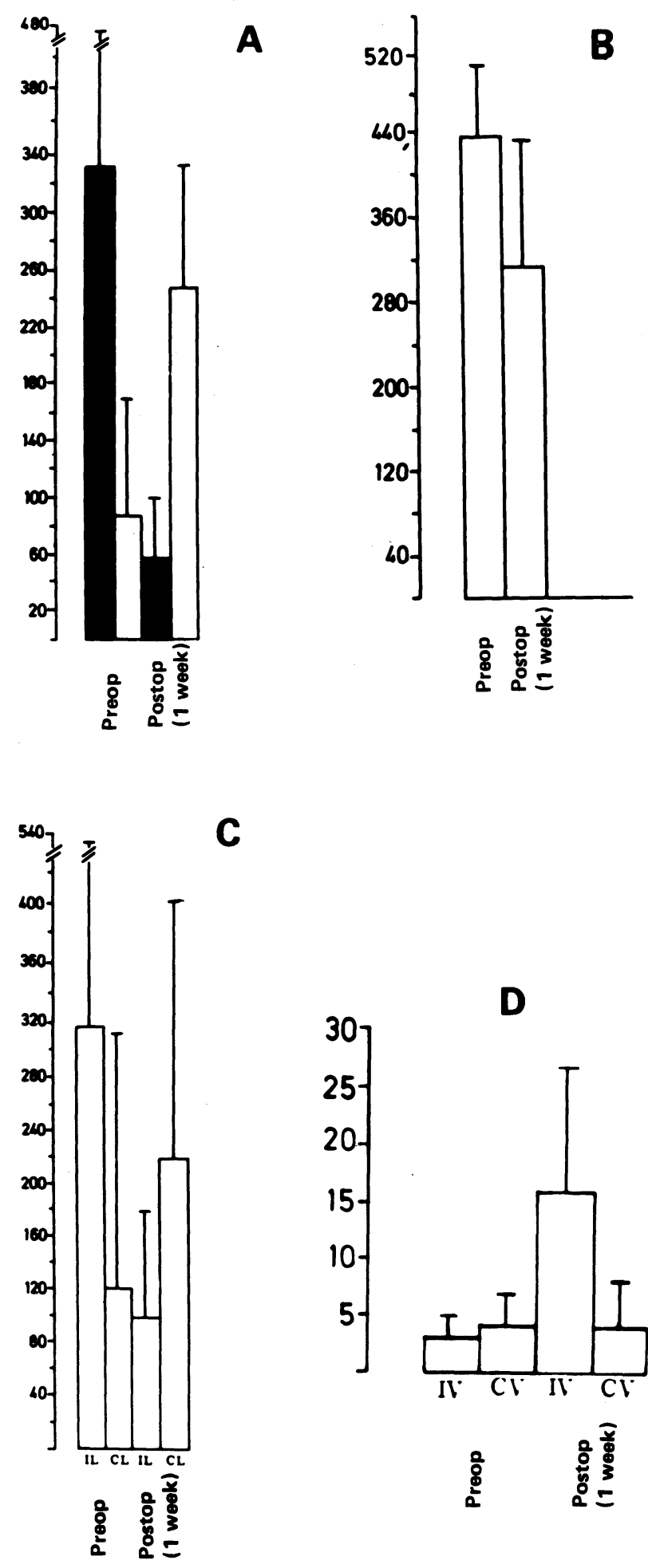

Figure 2. Group mean forepaw preferences (A) (stippled columns, preoperatively preferred paw), leverpresses (B), lever choice (C), and rotations (D), displayed by kainic-acid-lesioned rats in $50 \mathrm{~min}$ of testing in a two-lever Skinner box, before surgery and 1 week after surgery. Each bar represents one SD. (Abbreviations: Pre-op, before surgery; Post-op, after surgery; IL, ipsilateral lever; CL, contralateral lever; IV, ipsiversive rotation; $\mathrm{CV}$, contraversive rotation.) 
Table 2

Neurochemical Deficits in the Lesioned Hemisphere of Rats Sustaining Unilateral Kainate-Induced Damage of the Striatum Contralateral to the Predominantly Used Forepaw

\begin{tabular}{lccccc}
\hline & \multicolumn{5}{c}{ Percent Loss } \\
\cline { 2 - 6 } Subject & $\begin{array}{c}\text { Striatal } \\
\text { CAT }\end{array}$ & $\begin{array}{c}\text { Striatal } \\
\text { GAD }\end{array}$ & $\begin{array}{c}\text { Nigral } \\
\text { GAD }\end{array}$ & $\begin{array}{c}\text { "Limbic" "Limbic" } \\
\text { DA }\end{array}$ & NE \\
\hline A* & 80 & 70 & 47 & 63 & 68 \\
B & 44 & 65 & 56 & 17 & 19 \\
C & 72 & 91 & 0 & 2 & 0 \\
D & 53 & 46 & 36 & 34 & 24 \\
E & 73 & 27 & 0 & 50 & 26 \\
F & 49 & 67 & 34 & 5 & 0 \\
G & 85 & 64 & 47 & 57 & 40 \\
Mean & 63 & 60 & 29 & 28 & 18 \\
SD & 16 & 22 & 24 & 23 & 16 \\
\hline
\end{tabular}

*Because this subject suffered a huge reduction in mesolimbic ("Limbic") levels of catecholamines, it was excluded from all analyses of data relating to the behavioral effects of the lesion.

Also, mesolimbic NE was not consistently reduced on the lesion side; in fact, its level was higher on the lesion side in two subjects (one of the subjects with higher GAD activity in the ipsilateral substantia nigra plus one other subject).

Ipsilateral mesolimbic DA was, disappointingly, reduced to some extent in all the subjects, although the reduction was only $2 \%$ in one subject and $5 \%$ and $17 \%$ in two other subjects. One subject had a depletion of over $68 \%$ in mesolimbic DA.

Microscopic examination of the histological sections prepared from the brains of the subjects revealed no damage to structures posterior to the striatum in any of the animals. In all the subjects, the injected striatum was, of course, damaged. Figure 3 shows the appearance of the lesioned and unlesioned striata in one of them.

\section{EXPERIMENT 3}

\section{Materials and Methods}

Subjects. Six male Wistar albino rats, weighing between 180 and $200 \mathrm{~g}$ at the time of surgery, constituted the subjects for this study. When the animals arrived in the laboratory, they were housed in twos in $\mathrm{RCl}$ cages of North Kent Plastic Cages Ltd in a reverse-daylight room, as in Experiments 1 and 2. Food and water were constantly available, except that (1) food was removed for $48 \mathrm{~h}$ prior to $\mathrm{T}$-maze training in the preoperative phase or for $24 \mathrm{~h}$ prior to the postoperative data-gathering phase, and (2) water was not available during T-maze sessions.

Behavioral studies. When the animals first arrived in the laboratory, they were allowed 7 days to get used to the experimental housing conditions before behavioral studies involving them were begun. Then they were trained to run a T-maze for food. The stem of the maze was $90 \mathrm{~cm}$ long, its sides measured $90 \mathrm{~cm}$ from one end to the other, and the passage was $15 \mathrm{~cm}$ wide and $15 \mathrm{~cm}$ deep throughout.

Two data-gathering sessions of 10 runs each were given over the 2 consecutive days following the completion of training. Then the subjects underwent surgery. After surgery, they were allowed a test-free period of 7 days, and were then given two postoperative test sessions of 10 runs each over 2 consecutive days.

The categories of data recorded in the data-gathering sessions before and after surgery were: number of times a subject chose each arm of the maze and number of times a subject rejected each goalbox. A goalbox was adjudged rejected if a subject turned fully into the corresponding arm of the maze and then turned away and left without feeding, whether in the process the subject got as far as the goalbox or not. Side preferences and goal rejections were recorded with the help of a manually operated multichanneled counting device, each channel being used during any one session for only one kind of information, such as choice of left side or rejections of right goalbox.

Surgery. The surgical operations were the same as those adopted in Experiment 1, except that all the subjects in the present experiment received a dose of 6-OHDA in the MFB contralateral to the arm of the maze predominantly chosen in the preoperative datagathering session.

Verification of lesion. The subjects were sacrificed for the verification of the lesion 6 weeks after surgery. Left and right striatal and left and right mesolimbic samples were dissected out and assayed for DA and NE contents, as in Experiment 1.

\section{Results}

T-Maze behavior. Figure 4 depicts the effect of unilateral nigrostriatal lesions on the side preferences of the subjects in a T-maze. The reversal of the pat-
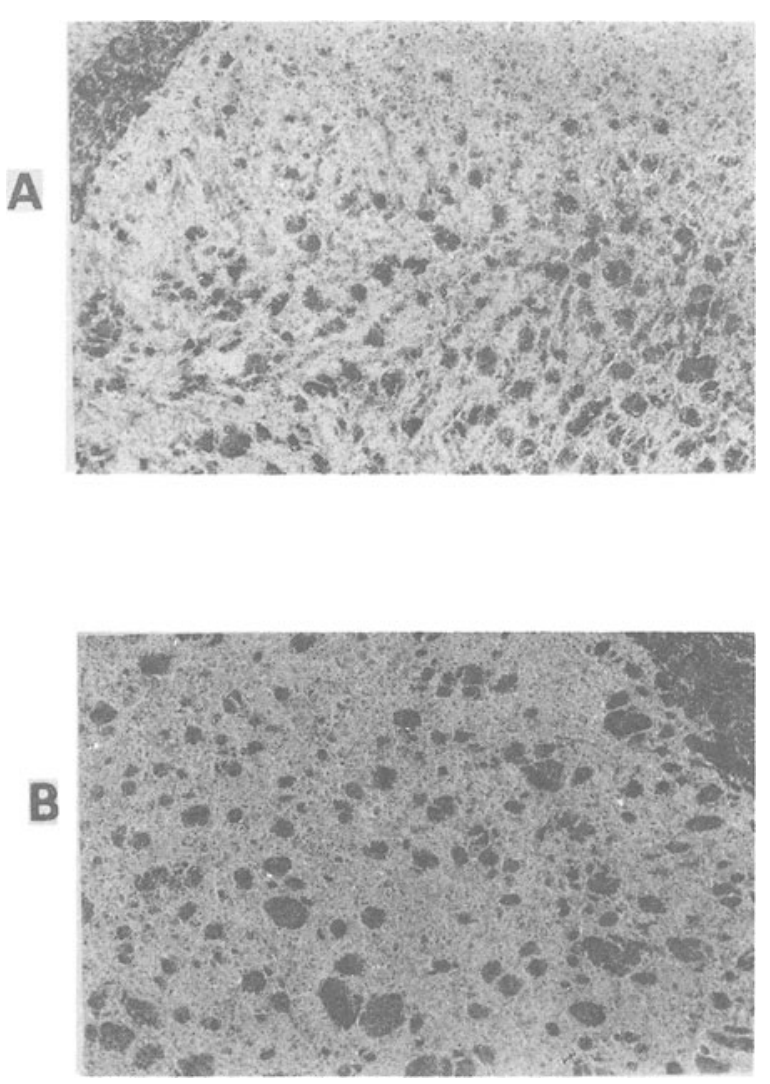

Figure 3. Microphotographs showing the striatum on each side of the brain of a rat given a unilateral intrastriatal injection of kainic acid. (A) Lesioned striatum. (B) Intact striatum. (Abbreviation: RCC, radiatio corporis callosi.) 

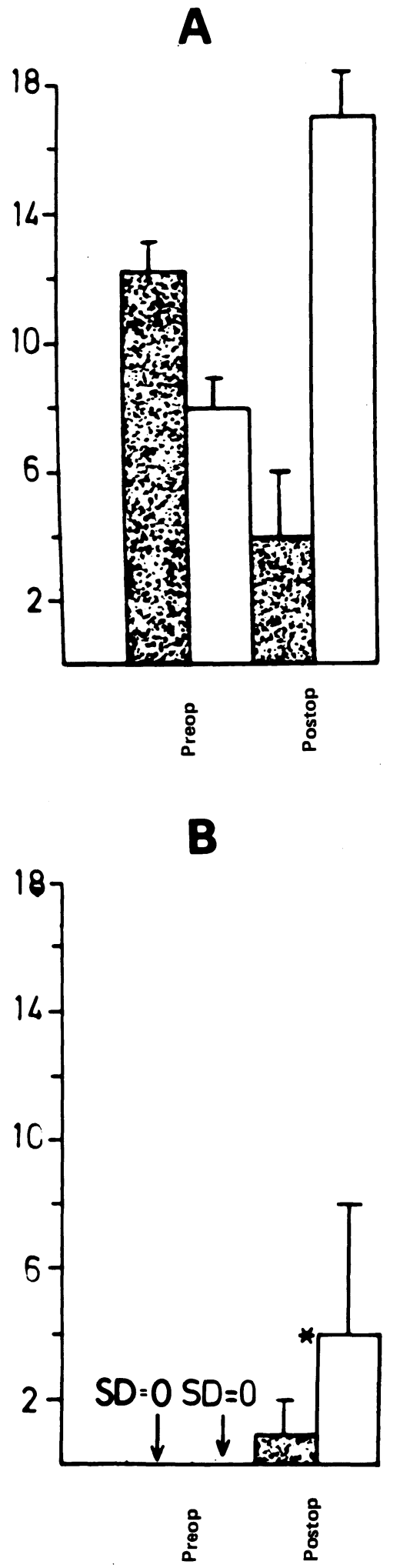

Figure 4. Group mean patterns of side preference and goal rejections displayed by rats in 20 trials in a $T$-maze before and after a unilateral 6-OHDA lesion of the MFB. Each bar represents one SD. (Abbreviations: Pre-op, before surgery; Post-op, after surgery.)
Table 3

Percent Catecholamine Reductions in the Lesioned Hemisphere of Rats Given a Unilateral 6-OHDA Microinjection into the MFB Opposite to the Preoperatively Preferred Side of a T-Maze

\begin{tabular}{ccccc}
\hline & \multicolumn{2}{c}{ Dopamine } & & \multicolumn{2}{c}{ Norepinephrine } \\
\cline { 2 - 4 } Subject & Striatum & Mesolimbic & Striatum & Mesolimbic \\
\hline A & 99 & 79 & 82 & 4 \\
B & 100 & 87 & 94 & 0 \\
C & 100 & 96 & 94 & 15 \\
D & 99 & 96 & 87 & 0 \\
E & 99 & 78 & 100 & 17 \\
F & 100 & 100 & 87 & 74 \\
\hline
\end{tabular}

tern of side preferences following surgery was dramatic in these animals, which were all lesioned on the side contralateral to the preoperative side preference.

At the postoperative test, the subjects circled frequently and only in the direction of the lesioned MFB. When leaving a goalbox, the subjects turned by the lesion side-never contraversively. In order to return to the startbox, the subjects often rotated again, also ipsiversively, at the junction; however, sometimes the subjects turned into the stem of the maze straight from the chosen side (contraversive turning), although when they did this they appeared to do so with difficulty.

The postoperative test saw also a marked increase in the number of times the subjects turned away from a goalbox without feeding (goal rejection). Nearly all the rejections were in respect to the goalbox in the preoperatively nonpreferred arm (side) of the maze.

Catecholamine assay results. The percent depletions of DA and NE in the striatum and the mesolimbic area of the lesioned hemisphere of the animals used in this study are shown in Table 3. From the table it can be seen that all the subjects sustained a striatal DA loss of $99 \%$ or more in the lesioned hemisphere; however, it may be noted that striatal $\mathrm{NE}$ and also mesolimbic DA and NE were depleted as well.

\section{DISCUSSION}

The results from Experiments 1 and 2 strongly suggest that the nigrostriatal DA system is involved in the sensorimotor control of behavior. As Figure 1 shows, rats trained to push a lever for food in an operant behavior situation and then given a 6-OHDA microinjection into the MFB contralateral to the preferred forepaw exhibit a reversal of forepaw preferences when tested postoperatively, whereas forepaw preferences are not affected in ipsilaterally lesioned animals or in controls. These results suggest that the contralateral forepaw is incapacitated selectively following a unilateral microinjection of 6-OHDA into the MFB, a suggestion supported by the observation that most of the lesioned animals tended to have their contralateral forelimb hanging 
limply down between the grids of the Skinner box floor during leverpressing.

In other words, whatever the important system that is damaged as a result of 6-OHDA microinjection to the MFB, it must be, like the pallidum (Levine et al., 1971), the entopeduncular nucleus (Levine \& Schwarzbaum, 1973), and the striatum (Hansing, Schwartzbaum, \& Thompson, 1968), part of a lateralized system controlling forelimb use.

As shown in Table 1, the MFB administration of 6-OHDA reduced catecholamine levels in the rostral regions of the brain. Most severely depleted were striatal and mesolimbic DA levels. In this regard, it should be noted that, although there are strong indications of nigrostriatal dopaminergic involvement in the sensorimotor control of behavior (Hansing et al., 1968; Marshall et al., 1974), the nucleus accumbens, which is a limbic structure with a rich supply of DA, appears to be involved in locomotor activity in rats (Fibiger, Fibiger, \& Zis, 1973; Jackson, Anden, \& Dahlstrom, 1975; Pijnenberg \& Van Rossum, 1973). It is not, therefore, possible, solely on the strength of the 6-OHDA experiment, to conclude that the disruption of the nigrostriatal DA pathway was or was not responsible for the observed incapacitation of the contralateral forelimb in animals sustaining a unilateral lesion of MFB. Disruption of the DA system supplying the mesolimbic area, or even of the NE system, which supplies the striatum and the origin and function of which are at present not clear, might conceivably be responsible for this effect.

The kainic acid experiment (Experiment 2) was designed primarily to deal with this interpretational difficulty. As Figure 2 shows, all the animals that suffered a kainate ablation of the neuronal perikarya intrinsic to the striatum on the side contralateral to the postoperatively preferred forepaw exhibited a reversal of forepaw preferences when tested postoperatively on an operant task acquired prior to surgery. This effect, which is precisely the same as that seen in animals with a 6-OHDA lesion of the MFB opposite to the preoperatively preferred forepaw (see Figure 1), is not likely to be due to extrastriatal damage, since histological examination of the brains of the kainic-acid-lesioned animals revealed no such damage in the thalamus and the pallidum, structures known to be involved in motor behavior.

Kainate lesion of the striatum has been reported to spare axon terminals and fibers of passage (Schwarcz $\&$ Coyle, 1977), and in the kainate lesion experiment presented here the reductions in mesolimbic DA were not as severe (see Table 2) as those observed in animals with a 6-OHDA lesion of the MFB (Table 1). In fact, some of the kainate-lesioned animals, which displayed an indisputable effect of lesion on forepaw use, showed less reduction in mesolimbic DA than did certain other subjects which had sustained a partial lesion of the MFB on the contralateral side and had displayed no effect on forepaw preferences. Therefore, the forelimb dysfunction observed following kainic acid lesion of the striatum could not have been due to disruption of mesolimbic and striatal DA supplies.

Kainate ablation of striatal intrinsic neuronal perikarya obviously disrupts the nigrostriatal DA system postsynaptically by removing the postsynaptic receptors of DA and/or other links in the chain of neural transmission of impulses reaching the striatum via the nigrostriatal DA pathway. The complementary use of both a direct (6-OHDA-induced) lesion of the nigrostriatal system and a kainate lesion procedure disrupting the nigrostriatal DA system postsynaptically appears therefore to have confirmed the involvement of this system in the sensorimotor control of behavior, although it does not necessarily show that sensorimotor performance would be unaffected by an extensive selective disruption of the system supplying DA to the mesolimbic area, notably the nucleus accumbens. This combined approach to the question of nigrostriatal dopaminergic involvement in sensorimotor control does demonstrate, furthermore, that the cardinal principle in this control is that of uninterrupted transmission of relevant nerve impulses on a network which includes, among other components, the pallidum, the entopeduncular nucleus, the striatum, and the nigrostriatal DA pathway.

Concerning the precise role of the nigrostriatal DA pathway in the sensorimotor control of behavior, it must be admitted that the present project has not determined that. Ranje and Ungerstedt (1977) have, however, proposed that the deficit which results from a lesion of the nigrostriatal DA system is "an interruption of sensory motor integration." In other words, the role of this pathway is neither purely sensory nor purely motor; rather, it serves to integrate stimuli with movement patterns. In the light of this hypothesis, it would appear that the sensorimotor dysfunction observed in the present investigations reflects a disruption of this integrative function of the nigrostriatal DA pathway.

That experimentally produced asymmetry between the nigrostriatal DA systems of the two hemispheres makes an animal rotate toward the less active of the two systems has been demonstrated by several authors (Anden et al., 1966; Arbuthnott \& Crow, 1971; Arbuthnott \& Ungerstedt, 1970; Zimmerberg \& Glick, 1975). In agreement with the results published by these authors is the observation presented in this paper that a unilateral lesion of the nigrostriatal DA pathway produces an enhancement of the number of rotations an animal performs during an operant behavior session and predisposes the animal to rotate only in the direction of the lesioned nigrostriatal DA pathway (see Figure 1).

In one of the experiments presented here, unilateral postsynaptic disruption of the nigrostriatal DA system, achieved by lesioning the striatum on one side with 
kainic acid, also produced a certain amount of increase in rotational behavior and some preference for the ipsiversive direction of circling (see Figure 2). However, the rats with a kainate lesion of the striatum performed many fewer rotations than those with a 6-OHDA lesion of the MFB over an identical period of time. Moreover, a unilateral kainate lesion of the striatum permitted some rats to perform contraversive as well as ipsiversive rotations, whereas the rotations performed at an identical time after surgery (i.e., 1 week postoperatively) by animals sustaining a unilateral 6-OHDA lesion of the MFB were exclusively ipsiversive. An explanation for these differences between the effects of the two kinds of unilateral damage on rotational behavior is not readily available. However, it is important to note that, whereas successful 6-OHDA microinjection into the MFB completely destroys the nigrostriatal DA pathway and cuts off DA supply to the striatum (see Table 1) without damaging noncatecholamine systems (Ungerstedt, 1968,1971 a) except for a small area of tissue necrosis at the site of injection (Butcher, 1975; Javoy, Soleto, Herbert, \& Agid, 1976), a kainate lesion of the striatum may or may not ablate all the DA postsynaptic receptor neurons, since a limited volume of the solution is used to prevent it from spreading outside the striatum. Thus, greater nigrostriatal asymmetry is produced by a unilateral 6-OHDA lesion of the MFB than by a unilateral kainate lesion of the striatum, and it should not be very surprising that lesion effect on rotational behavior is more pronounced following the former than following the latter surgical procedure.

As well as rotational behavior, side preference in a T-maze has been reported to be related to asymmetry between the nigrostriatal DA systems of the two hemispheres (Rothman \& Glick, 1976; Zimmerberg, 1975; Zimmerberg et al., 1974). In one of these studies (Rothman \& Glick, 1976), a unilateral electrolytic caudate lesion produced preference for the ipsilateral arm of the maze, and lesions localized in certain parts of the striatum were more effective than lesions placed in other parts of the same structure. Rothman and Glick (1976) concluded that reduced activity in the nigrostriatal DA system on the lesion side was responsible for the ipsilateral side preference displayed by those of their rats sustaining an effective unilateral caudate lesion. Their conclusion is confirmed by the results of the T-maze experiment here presented (see Figure 4), in which a unilateral 6-OHDA lesion of the nigrostriatal DA pathway on the side opposite to the preferred arm of a $\mathrm{T}$-maze produced a dramatic reversal of side preference.

Although the effect of a unilateral kainate lesion of the striatum on T-maze behavior was not investigated in the present project, it seems likely that such a lesion will also produce an ipsilateral side preference, provided the DA postsynaptic receptor neurons in the striatum are decimated or completely ablated.
In the present project, neither a unilateral 6-OHDA lesion of the nigrostriatal DA pathway nor a unilateral kainate lesion of the striatum produced a preference for the ipsilateral lever in a two-lever operant behavior situation. The former surgical procedure produced no consistent effect on lever preference, and the latter procedure actually appears to have led to increased preference for the contralateral lever. These observations are at variance with the report by Glick and Jerussi (1974) that rats prefer the lever on the same side as the striatum with the lesser supply of DA when tested in a two-lever operant-behavior situation. The discrepancy between the present author's results, on the one hand, and the results obtained by Glick and Jerussi, on the other, regarding lever preference is difficult to explain, although there is the possibility that important differences exist between the operant behavior boxes used. Anyway, the observation that a reversal of side preference in a $\mathrm{T}$-maze following a unilateral lesion of the nigrostriatal pathway is associated with increased goal rejections, particularly on the side less used prior to surgery, may be relevant to the interpretation of the present author's results with respect to lever preference in a two-lever Skinner box. Thus, goal preference, as distinct from side preference, in a $\mathrm{T}$-maze seems to represent a dimension of spatial behavior which is not determined by asymmetry between the nigrostriatal systems of the brain in the way that side preference is. The dimension of spatial behavior represented by goal preference in a T-maze may be defined in terms of locating objects in space and would include lever preference in a two-lever Skinner box. If this hypothesis is correct, it should not be surprising that a unilateral nigrostriatal pathway lesion failed to produce an ipsilateral lever preference in the present project, even though such a lesion produces ipsiversive rotation and ipsilateral side preference.

The observation that a unilateral kainate lesion of the striatum produced a preference for the contralateral lever is even more difficult to explain. However, a hypothesis that may be worth considering is that the striatum contains neurons which are concerned with a kind of spatial preference based on the active location of objects in space. If such neurons exist in the striatum, the indication from the relevant experiment herein presented (see Figure 2) is that they function ipsilaterally; in other words, it appears that neurons intrinsic to the left striatum would be primarily concerned with the area of space to the left, whereas right striatal neurons would focus more on the right.

\section{REFERENCES}

Anden, N.-E., Dahlstrom, A., Fuxe, K., Larsson, K., Olson, L., \& UNGerstedt, U. Ascending monoamine neurons to the telencephalon and diencephalon. Acta Physiologica Scandinavica, 1966, 67, 313-326.

Arbuthnot, G. W., \& Crow, T. J. Relation of contraversive 
turning to unilateral release of dopamine from the nigrostriatal pathway in rats. Experimental Neurology, 1971, 30, 484-491.

Arbuthnot, G. W., \& Ungerstedt, U. Locomotor behaviour after electrical stimulation of dopamine-containing neurons. Acta Physiologica Scandinavica, 1977, 77, Suppl. 330, 117.

Bedard, P., Larochelle, L., Parent, A., \& Poirier, L. J. The nigrostriatal pathway. A correlative study based on neuroanatomical and neurochemical criteria in the cat and the monkey. Experimental Neurology, 1969, 25, 365-377.

Butcher, L. L. Degenerative processes after punctate intracerebral administration of 6-hydroxydopamine. Journal of Neural Transmission, 1975, 37, 189-208.

Carlsson, A., Lindqvist, M., Magnusson, T., \& Waldeck, B. A. On the presence of 3-hydroxytyramine in brain. Science, $1958,127,471$.

Carlsson, A., \& Waldeck, B. A. Fluorometric method for the determination of dopamine (3-hydroxytyramine). Acta Physiologica Scandinavica, 1958, 44, 293-298.

Christie, J. E., \& Crow, T. J. Turning behaviour as an index of the action of amphetamines and ophedrines on central dopamine-containing neurons. British Journal of Pharmacology, 1971, 43, 658-667.

Coyle, J. T., \& Henry, D. Catecholamines in fetal and newborn rat brain. Journal of Neurochemistry, 1973, 21, 61-67.

Donaldson, I. M., Dolphin, A., Jenner, P., Marsden, C. D., \& Pycock, C. Contraversive circling behaviour produced by unilateral electrolytic lesions of the ventral noradrenergic bundle mimicking the changes seen with unilateral electrolytic lesions of the locus coeruleus. Journal of Pharmacy and Pharmacology, 1976, 28, 329-331.

Drummond, R. J., \& Phillips, A. T. L-glutamic acid decarboxylase in non-neural tissues of the mouse. Journal of Neurochemistry, 1974, 23, 1207-1213.

Fibiger, H. C., Fibiger, H. P., \& Zis, A. P. Attenuation of amphetamine induced motor stimulation and stereotypy by 6-hydroxydopamine in the rat. British Journal of Pharmacology, 1973, 47, 683-692.

Fonnum, F. A. Rapid radiochemical method for the determination of choline acetyltransferase. Journal of Neurochemistry, 1975, 24, 407-409.

Glick, S. D., \& Jenussi, T. P. Spatial and paw preferences in rats: Their relationship to rate-dependent effects of D-amphetamine. Journal of Pharmacology and Experimental Therapeutics, 1974, 188, 714-725.

Hansing, R. A., Schwartzbaum, J. S., \& Thompson, J. B. Operant behavior following unilateral and bilateral caudate lesions in the rat. Journal of Comparative and Physiological Psychology, 1968, 66, 378-388.

Jackson, D. M., Anden, N.-E., \& Dahlstrom, A. A functional effect of dopamine in the nucleus accumbens and in some other dopamine-rich parts of the rat brain. Psychopharmacologia, 1975, 45, 139-149.

Javoy, F., Soleto, C., Herbert, A., \& AgID, Y. Specificity of dopaminergic neuronal degeneration induced by intracerebral injection of 6-hydroxydopamine in the nigro-striatal dopaminergic system. Brain Research, 1976, 201-215.

Kluver, H., \& Barrera, E. A. A. A method for the combined study of cells and fibres in the nervous system. Journal of Neuropathology and Experimental Neurology, 1953, 12, 400-403.

Levine, M. S., Ferguson, N., Kreinick, C. J., Gustafson, J. N., \& Schwarzbaum, J. S. Sensorimotor dysfunctions and aphagia and adipsia following pallidal lesions in rats. Journal of Comparative and Physiological Psychology, 1971, 77, 282-293.
Levine, M. S., \& Schwarzbaum, J. S. Sensorimotor functions of the striatopallidal system and the lateral hypothalamus and consummatory behavior in rats. Journal of Comparative and Physiological Psychology, 1973, 85, 615-635.

LJUNGBERG, T., \& UNGERSTEDT, U. Sensory inattention produced by 6-hydroxydopamine induced degeneration of ascending dopamine neurons in the brain. Experimental Neurology, 1976, 53, 585-600.

Marshall, J. F., Richardson, J. S., \& Teitelbaum, P. Nigrostriatal bundle damage and the lateral hypothalamic syndrome. Journal of Comparative and Physiological Psychology, 1974, 87, 808-830.

Moore, R. Y., Bhatnager, R. K., \& Heller, A. Anatomical and chemical studies of a nigro-neostriatal projection in the cat. Brain Research, 1971, 30, 119-135.

Palkovitz, M., Brownstein, M., Seavedra, J. M., \& AXELrod, J. Norepinephrine and dopamine content of hypothalamic nuclei of the rat. Brain Research, 1974, 77, 137-149.

Pijnenberg, A. J. J., \& Van Rossum, J. M. Stimulation of locomotor activity following injection of dopamine into the nucleus accumbens. Journal of Pharmacy and Pharmacology, 1973, 25, 1003-1005.

Pycock, C. J., Donaldson, I. M., \& Marsden, C. D. Circling behaviour produced by unilateral lesions in the region of the locus coeruleus in rats. Brain Research, 1976, 97, 317-329.

RANJE, C., \& UNGERSTEDT, U. Lack of acquisition in dopamine denervated animals tested in an underwater Y-maze. Brain Research, 1977, 134, 95-111.

Rothman, A. H., \& GLICK, S. D. Differential effects of unilateral and bilateral caudate lesions on side preference and passive avoidance behavior in rats. Brain Research, 1976, 118, 369.

Schwarcz, R., \& Coyle, J. T. Striatal lesions with kainic acid: Neurochemical characteristics. Brain Research, 1977, 127, 235-249.

UNGERSTEDT, U. 6-hydroxydopamine induced degeneration of central monoamine neurons. European Journal of Pharmacology, 1968, 5, 107-110.

UNGERSTEDT, U. Histochemical studies on the effects of intracerebral and intraventricular injections of 6-hydroxydopamine on monoamine neurons in the rat brain. In T. Malmfors \& $\mathbf{H}$. Thoenen (Eds.), 6-Hydroxydopamine and catecholamine neurons. Amsterdam: North-Holland, 1971. (a)

UNGERSTEDT, U. Postsynaptic supersensitivity after 6-hydroxydopamine induced degeneration of the nigrostriatal dopamine system. Acta Physiologica Scandinavica, 1971, Suppl. 367, 69-93. (b)

Urquhart, N., Perry, T. L., Hansen, S., \& Kannedy, J. GABA content and glutamic acid decarboxylase activity in brain of Huntington's chorea patients and control subjects. Journal of Neurochemistry, 1975, 24, 1071-1075.

Zimmerberg, B. Nigrostriatal asymmetry and associative functions of the striatum. Unpublished doctoral dissertation, City University of New York, 1975.

ZimmerberG, B., \& Glick, S. D. Changes in side preference during unilateral electrical stimulation of the caudate nucleus in rats. Brain Research, 1975, 86, 335-338.

Zimmerberg, B., Glick, S. D., \& Jerussi, T. P. Neurochemical correlate of a spatial preference in rats. Science, 1974, 185, 623-625.

(Manuscript received January 19, 1981; revision accepted for publication August 4, 1981.) 